\title{
Excess Body Weight Loss After Laparoscopic Gastric Plication
}

\author{
Mohamed Salah Abdelhamid ${ }^{1}$, Ayman Hamdi Abouleid ${ }^{1}$, Mohamed Hasan Abdelmola ${ }^{1}$, \\ Ahmed Mohamed Rashad ${ }^{1}$, Mahmoud Ahmed Negida ${ }^{2}$, Ahmed Zaky Gharib ${ }^{3}$ \\ ${ }^{1}$ Surgery Department, Bani-Suef Faculty of Medicine, Bani-Suef University, Bani-Suef, Egypt \\ ${ }^{2}$ Surgery Department, Kasr Elaini Faculty of Medicine, Cairo University, Cairo, Egypt \\ ${ }^{3}$ Surgery Department, October 6th Faculty of Medicine, October 6th University, Giza, Egypt \\ Email address: \\ Mohamedsalah_2000@hotmail.com (M. S. Abdelhamid), Ayman_abouleid@hotmail.com (A. H. Abouleid), \\ dr-m_hasan@outlook.com (M. H. Abdelmola), rashad-77393@yahoo.com (A. M. Rashad), Dr-negida@yahoo.com (M. A. Negida), \\ dr-ahmedzaki@yahoo.com (A. Z. Gharib)
}

\section{To cite this article:}

Mohamed Salah Abdelhamid, Ayman Hamdi Abouleid, Mohamed Hasan Abdelmola, Ahmed Mohamed Rashad, Mahmoud Ahmed Negida, Ahmed Zaky Gharib. Excess Body Weight Loss After Laparoscopic Gastric Plication. Advances in Surgical Sciences.

Vol. 4, No. 2, 2016, pp. 6-8. doi: 10.11648/j.ass.20160402.11

Received: February 25, 2016; Accepted: March 5, 2016; Published: March 22, 2016

\begin{abstract}
Gastric plication was selected for cases with potential for continuous diet and exercise after operation. The appetite of patients decreased after operation due to total gastric volume restriction. The study aimed at finding if it is worth or not to plicate in relation to excess body weight loss (EBWL). 47 patients were enrolled in the study between May 2014 till Nov 2015 with follow up till Nov 2015 with body mass index (BMI) of 38 -65. Laparoscopic gastric placation (LGP) was done to all using two rows of continuous o prolene thread aided with harmonic scalpel. BMI and EBW were calculated prior to LGP then EBWL was estimated at six months and one year. The following scale was applied regarding EBWL $0-14 \%$ failure, $15 \%$ $-39 \%$ poor, $40 \%-69 \%$ medium, $70 \%$ or more excellent outcome, while regain of $10 \%$ or more marked as regain. We had one (2.1\%) deplication, six $(12.7 \%)$ regained weight, two $(4.2 \%)$ failures, fourteen $(29.7 \%)$ poor, sixteen $(34 \%)$ medium outcome and eight (17\%) with excellent outcome regarding EBWL. We concluded that it is very difficult to roll out LGP, we found it is worth to do LGP although only $17 \%$ got excellent EBWL and 34\% got medium EBWL which was acceptable to those patients which represent more than $50 \%$ of our patients. This acceptance was supported by the low cost, safety and the potential reversibility of the procedure.
\end{abstract}

Keywords: LGP, EBWL, BMI

\section{Introduction}

Restrictive methods (RM) are the most conservative bariatric surgery. Malabsorptive method has longer effect on weight loss but the risk of late complications due to vitamin deficiency and anemia is noticeable [1]. Gastric plication was selected for cases with potential for continuous diet and exercise after operation [2]. The appetite of patients decreased after operation due to total gastric volume restriction. [3]. The price of hospitalization and instruments used in gastric plication was $2000 \$$, while gastric bypass would cost $4500 \$$ and sleeve or banding $4000 \$$ [4].

The complications were not more than other methods; and costs were far less than any other bariatric surgery. The final result of weight loss was almost the same as other restrictive techniques [5]. However, the wide range of weight loss resulted from different methods of restrictive bariatric surgery means the main reason of weight loss is not just a specific technique; but also could be mostly the patient's motivation to keep appropriate lifestyle [6]. Based on endoscopy and mucosal biopsy and chyme analysis in randomized cases and according to reoperation (due to gallstone or appendicitis), plicated fold showed shrinkage due to muscular atrophy, but the mucosa was normal. Shrinkage of plicated fold and extension of elastic gastric wall were two main factors for limited effective time of 
plication (4 years) [7]. Replication was easier than plication, because release of greater curvature had been done before, adhesions of liver or omentum to stomach were loose and resuturing over dilated plicated stomach was something like the second row suturing in plication method [8]. In vertical banded gastroplasty (VBG) the risk of reflux, leak, blow out of stomach, regain and foreign body reaction would be highly observed [4]. Complications secondary to sleeve gastrectomy including leakage, disruption and malabsorption is about $10 \%$ [9]. Effective time of all restrictive methods is limited to 3 to 4 years, so risk of regain is the problem of all kinds of bariatric surgeries. In duodenal switch (DS) there is a significant mal-absorptive component, so the patient must be closely monitored for nutritional deficiencies. These patients are at greater risk of chronic diarrhea, more foul smelling stools and flatus. In gastric bypass because it includes resection, anastomosis and malabsorption, the risk of mortality and morbidity are $1 \%$ and up to $15 \%$ respectively [10]. There is about $10 \%$ to $20 \%$ of emergency band removal (happening in wide range of time after surgery) in gastric banding operation because of balloon leakage, band erosion/migration, deep infection and reaction to foreign body [11].

\section{Patients and Methods}

Forty seven patients were enrolled in the study between May 2014 till Nov 2015 with follow up till Nov 2015 with BMI of 38 - 65. LGP was done to all using two rows of continuous o prolene thread aided with harmonic scalpel. BMI and EBW were calculated prior to LGP then EBW Loss was estimated at six months and one year. The following scale was applied regarding EBW loss 0- $14 \%$ failure, 15\%$39 \%$ poor, $40 \%-69 \%$ medium, $70 \%$ or more excellent outcome, while regain of $10 \%$ or more marked as regain.

\section{Results}

We had one $(2.1 \%)$ deplication, six $(12.7 \%)$ regained weight, two (4.2\%) failures, fourteen $(29.7 \%)$ poor, sixteen (34\%) medium outcome and eight (17\%) with excellent outcome regarding EBWL.

Table 1. Demographic data of the patients.

\begin{tabular}{lll}
\hline Gender & Females 35 & Males 12 \\
\hline Age & $19-50$ years & Mean 33.9 years \\
Preoperative BMI & $40-62.8 \mathrm{Kg} / \mathrm{m}^{2}$ & Mean $51.09 \mathrm{~kg} / \mathrm{m}^{2}$ \\
\hline
\end{tabular}

Operative time ranged from 120 minutes to 210 minutes, with a mean of 174 minutes. Regarding intra-operative mishaps repeated cutting of the thread in one case. All patients were discharged from the hospital after an average of 2.25 days (range 2 to 3 days). All patients were done laparoscopically and completed with no conversion, no blood transfusion needed in all cases.

\section{Discussion}

Long term results depend mainly on the extent of motivation to keep on corrected lifestyle. Restrictive effect of surgery takes until 4 years and then enlargement of stomach up to $250 \mathrm{cc}$ is enough to induce regain

As the main group of cases were young females and they were interested in plication due to very low complication rate, we think the main factor for increasing motivation of these patients was social factors like marriage; so that after some time of operation these social factors have been solved and patients' motivations has decreased.

As the effects of all restrictive methods are almost the same, the best method is the one with the least risk of complication. Gastric plication has the least rate of reoperation which is due to $1 \%$ technical (in the first week) and less than $0.2 \%$ late complications. It is noteworthy in comparison to other restrictive methods like gastric banding or sleeve gastrectomy

Talepour stated that laparoscopic gastric plication is as effective as other restrictive methods to lose weight. Its advantages include: easy follow up, no foreign body, much less cost, low complications $(0.6 \%)$, low reoperation $(1 \%)$, only $31 \%$ regain up to 12 years follow up, easy plan for regain group, psychological advantage due to independency to surgeon and sense of normal physiology and anatomy without any resection. This method is reversible if needed and also does not prohibit complementary malabsorptive methods (2 stage operation) in cases with insufficient weight loss [12].

Shen stated one year after surgery, the mean \%EBWL was $58.8 \pm 16.7 \%(\mathrm{n}=11)$ in the LGP group [13], this goes hand in hand with our outcome as we had 51\% EBWL at the end of the first year. This also in accordance with Yun et al who stated that the percentage of EBWL for LGP varied from $31.8 \%$ to $74.4 \%$ with follow-up from 6 months to 24 months [14].

Special hormones of stomach (Ghrelin, Leptin etc.) make an appetite balance in normal condition. Although the effect of ghrelin on appetite is remarkable, balance between gastric hormones and appetite was not changed after sleeve gastrectomy [15].

Losing appetite is related to decreased intraluminal space and high intraluminal pressure. This mechanism is more noticeable in gastric plication. When intraluminal space increases during time, appetite increases as well regardless of surgical technique used.

To sum it up, advantages of LGP over other restrictive methods are as follows. First, the patient is independent postoperatively with easy follow up; which means the patient is free from any obligatory post-operative procedures e.g. balloon size regulation in gastric banding. No foreign body reaction is the next advantage of LGP as only two or three prolene or nylon threads are used with no use of mesh or band.

Owen Haskins study showed EBWL at the range of 50\% at six months and $60 \%$ on 12 months [16] more or less exactly 
with ours as we got $51 \%$ EBWL at the end of the first year (34\% medium outcome and $17 \%$ excellent outcome).

Moreover, less cost due to no need for stapler or band and short period of postoperative hospitalization are very important factors for patients. Less complication such as leakage, infection or erosion should be noted since this method is the most conservative procedure among other bariatric surgeries with no resection or anastomosis.

In case when the patient is not tolerating the procedure deplication is reversible during first 6 weeks just by cutting the suture lines and easily releasing the mild adhesions. Reversion of LGP later is difficult and it needs tense fibrotic tissue dissection. It can be followed by adding malabsorptive method as a second stage operation in cases with insufficient weight loss without any change to previous surgery.

As there is no usage of stapler and resection of greater curvature, LGP is less invasive and more conservative with reversible potency and less risk of complications such as leakage and bleeding which if happened is very costly to manage.

\section{Conclusion}

It is very difficult to roll out LGP, we found it is worth to do LGP although only 17\% got excellent EBWL and 34\% got medium EBWL which were acceptable to those patients which represent more than $50 \%$ of our patients. This acceptance was supported by the low cost, safety and the potential reversibility of the procedure.

\section{References}

[1] Crea N, Pata G, Di Betta E, Greco F, Casella C, Vilardi A, Mittempergher F. Long-term results of biliopancreatic diversion with or without gastric preservation for morbid obesity. Obes Surg.2011; 21: 139-145. doi: 10.1007/s11695010-0333-6. [PubMed] [Cross Ref].

[2] Campbell J, McGarry LA, Shikora SA, Hale BC, Lee JT, Weinstein MC. Cost-effectiveness of laparoscopic gastric banding and bypass for morbid obesity. Am J Manag Care. 2010; 16: e174-e187. [PubMed].

[3] Talebpour M, Amoli BS. Laparoscopic total gastric vertical plication in morbid obesity. Journal of laparoendoscopic \& advanced surgical techniques Part A. 2007; 17: 793-798. doi: 10.1089/lap.2006.0128. [PubMed] [Cross Ref].

[4] Scozzari G, Toppino M, Famiglietti F, Bonnet G, Morino M. 10-year follow-up of laparoscopic vertical banded gastroplasty: good results in selected patients. Ann Surg. 2010; 252: 831-839. doi: 10.1097/SLA.0b013e3181fd35b0. [PubMed] [Cross Ref].

[5] Ramos A, Galvao Neto M, Galvao M, Evangelista LF, Campos JM, Ferraz A. Laparoscopic greater curvature plication: initial results of an alternative restrictive bariatric procedure. Obes Surg.2010; 20: 913-918. doi: 10.1007/s11695-010-0132-0. [PubMed] [Cross Ref].
[6] Pujol Gebelli J, Garcia Ruiz de Gordejuela A, Casajoana Badia A, Secanella Medayo L, Vicens Morton A, Masdevall Noguera C. Laparoscopic Gastric Plication: a new surgery for the treatment of morbid obesity. Cir Esp. 2011; 89(6): 356361. doi: 10.1016/j.ciresp.2011.02.005. [PubMed] [Cross Ref].

[7] Brethauer SA, Harris JL, Kroh M, Schauer PR. Laparoscopic gastric plication for treatment of severe obesity. Surg Obes Relat Dis. 2011; 7: 15-22. doi: 10.1016/j.soard.2010.09.023. [PubMed] [Cross Ref].

[8] Muller MK, Wenger C, Schiesser M, Clavien PA, Weber M. Quality of life after bariatric surgery--a comparative study of laparoscopic banding vs. bypass. Obes Surg. 2008; 18: 15511557. doi: 10.1007/s11695-008-9522-y. [PubMed] [Cross Ref].

[9] D’Hondt M, Vanneste S, Pottel H, Devriendt D, Van Rooy F, Vansteenkiste F. Laparoscopic sleeve gastrectomy as a singlestage procedure for the treatment of morbid obesity and the resulting quality of life, resolution of comorbidities, food tolerance, and 6-year weight loss. Surg Endosc. 2011; 25(8): 2498-2504. doi: 10.1007/s00464-011-1572-x. [PubMed] [Cross Ref].

[10] Pontiroli AE, Morabito A. Long-term prevention of mortality in morbid obesity through bariatric surgery. a systematic review and meta-analysis of trials performed with gastric banding and gastric bypass. Ann Surg. 2011; 253: 484-487. doi: 10.1097/SLA.0b013e31820d98cb. [PubMed] [Cross Ref].

[11] Strain GW, Gagner M, Pomp A, Dakin G, Inabnet WB, Hsieh J, Heacock L, Christos P. Comparison of weight loss and body composition changes with four surgical procedures. Surg Obes Relat Dis.2009; 5: 582-587. doi: 10.1016/j.soard.2009.04.001. [PubMed] [Cross Ref].

[12] Mohammad Talebpour, ${ }^{1}$ Seyed Mohammad Kalantar Motamedi, ${ }^{1,2}$ Atieh Talebpour, ${ }^{1}$ and Hamed Vahidi ${ }^{1}$, Twelve year experience of laparoscopic gastric plication in morbid obesity: development of the technique and patient outcomes Ann Surg Innov Res. 2012; 6: 7. Published online Aug 22, 2012. doi: 10.1186/1750-1164-6-7 PMCID: PMC3444326.

[13] Shen D, Ye H, Wang Y, Ji Y, Zhan X, Zhu J, Li W Comparison of short-term outcomes between laparoscopic greater curvature plication and laparoscopic sleeve gastrectomy. Surg Endosc. 2013 Aug; 27(8): 2768-74. doi: 10.1007/s00464-013-2805-y. Epub 2013 Feb 27.

[14] Yun Ji, M. D. Yuedong Wang, M. D., F. A. C. S. Jinhui Zhu, M. D. Dijian Shen, M. D. A systematic review of gastric plication for the treatment of obesity Published Online: December $16,2013 \quad$ DOI: http://dx.doi.org/10.1016/j.soard.2013.12.003.

[15] Langer FB, Reza Hoda MA, Bohdjalian A, Felberbauer FX, Zacherl J, Wenzl E, Schindler K, Luger A, Ludvik B, Prager G. Sleeve gastrectomy and gastric banding: effects on plasma ghrelin levels. Obes Surg.2005; 15: 1024-1029. doi: 10.1381/0960892054621125. [PubMed] [Cross Ref].

[16] Owen Haskins Abdelbaki TN, Huang CK, Ramos A, Neto MG, Talebpour M, Saber AA Gastric plication for morbid obesity: a systematic review Obes Surg. 2012 Oct; 22(10): 1633-9. doi: 10.1007/s11695-012-0723-z. 